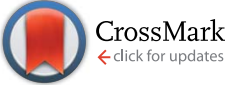

Cite this: RSC Adv., 2017, 7, 16861

\title{
Expanding analytical tools for characterizing ultrasmall silica-based nanoparticles
}

\begin{abstract}
B. Yoo, ${ }^{\star a b}$ K. Ma, $^{c}$ U. Wiesner ${ }^{\star c}$ and M. Bradbury ${ }^{\star a d}$
$C^{\prime}$ dots are fluorescent inorganic-organic hybrid nanoparticles synthesized in water comprised of a silica core with a covalently embedded near infrared dye, and a polyethylene glycol (PEG) outer layer. $C^{\prime}$ dots containing the integrin specific ligand, cycloRGDyC, are the first of their kind particles approved for human clinical trials. In the continued clinical development of these nanoparticles, high-resolution analytical approaches are needed. Here we investigate the use of reversed phase high performance liquid chromatography (RP-HPLC) to analyze cycloRGDyC-Cy5- $C^{\prime}$ dots. Given the stability and proteinlike size, we reasoned that these nanoparticles would be compatible under RP-HPLC conditions typically used to characterize peptides and proteins. Our results show that RP-HPLC provides excellent resolution, showing significant heterogeneity of these nanoparticles. $C^{\prime}$ dots also exhibit unusual peak profiles where RP-HPLC chromatogram peak shapes change from run to run, possibly due to the conformational heterogeneity or charge distribution of the particle surface due to the PEG groups. In addition we describe a novel thiol-mediated release of $C^{\prime}$ dot ligands to directly estimate cycloRGDyC by exposing the particles to organic thiols. Ligand release is presumably afforded by a reverse Michael reaction mechanism.
\end{abstract}

Received 1st February 2017
Accepted 7th March 2017

DOI: $10.1039 / c 7 r a 01349 c$

rsc.li/rsc-advances

spectral properties of particles, respectively., ${ }^{8,9}$ More recently, chromatographic approaches, such as gel permeation/filtration or size exclusion chromatography (GPC or SEC), have also become integrated into the workflow of nanoparticle characterization and purification..$^{9-11}$

Our research has been focused on the clinical development of specific types of ultrasmall (i.e. sizes below $10 \mathrm{~nm}$ ) fluorescent silica nanoparticles, known as $\mathrm{C}^{\prime}$ dots (Fig. 1). $\mathrm{C}^{\prime}$ dots are synthesized in water as opposed to alcoholic solutions in earlier efforts leading to $\mathrm{C}$ dots. ${ }^{12} \mathrm{C}^{\prime}$ dots are inorganic-organic hybrids comprised of a silica core and a polyethylene glycol (PEG) shell. ${ }^{10,13}$ For medical applications, embedded within the core are near infrared dyes (e.g., Cy5 or Cy5.5), which results in dramatically enhanced optical properties over the free dye as a result of the rigid silica matrix, ${ }^{10,14,15}$ while the PEG surface is partially functionalized with targeting peptides that can further be labeled with radioisotopes. ${ }^{16}$ Consequently, $\mathrm{C}^{\prime}$ dots have been shown to be clinically viable for dual-modality PET-optical imaging, and are a first-in-kind particle probe approved for human use. ${ }^{5}$ Additionally the stability, flexible surface chemistry, and particularly the small size of these particles - which affords good tissue penetration and predictable clearance profiles $^{17}$ - make them ideal candidates as drug delivery vehicles, an application which is currently being explored..$^{18}$

Characterization of $\mathrm{C}^{\prime}$ dots have included the standard regiment of approaches as listed above: UV-vis absorption spectroscopy, DLS, zeta-potential, TGA, TEM, and GPC. ${ }^{10}$ Addi-

tionally, the embedded dyes of $\mathrm{C}^{\prime}$ dots have enabled the use of

\footnotetext{
Department of Radiology, Sloan Kettering Institute for Cancer Research, New York New York 10065, USA. E-mail: yoob@mskcc.org; bradburm@mskcc.org ${ }^{b}$ Department of Chemistry, Hunter College, New York, New York 10065, USA ${ }^{c}$ Department of Materials Science \& Engineering, Cornell University, Ithaca, New York 14853, USA. E-mail: ubw1@cornell.edu

${ }^{d}$ Molecular Pharmacology Program, Sloan Kettering Institute for Cancer Research, New York, NY 10065, USA
}

Advances in nanotechnology have largely been driven by the development of novel materials with interesting properties emerging at the nanoscale. In biomedical research, nanoparticles in particular, have garnered attention largely due to
their physical attributes - discrete and relatively stable particles in the nanometer size regime with at least one novel/superior property. ${ }^{1,2}$ Their potential utility in biomedical applications, now being realized as a number of products have reached the cinical stage. ${ }^{1-5}$ nanoparticles as a viable clinical product is the arduous and complex process to rigorously assess their stability, toxicity, and particle morphologies, preclinical characterization of nanoparticles has largely relied on techniques used in the field of materials and polymer research, for example, UV-vis absorption spectroscopy, dynamic light scattering (DLS), zeta-potential, thermogravimetric analysis (TGA), or transmission electron microscopy (TEM), etc. These tools have provided necessary information regarding the size, shape, composition, and 


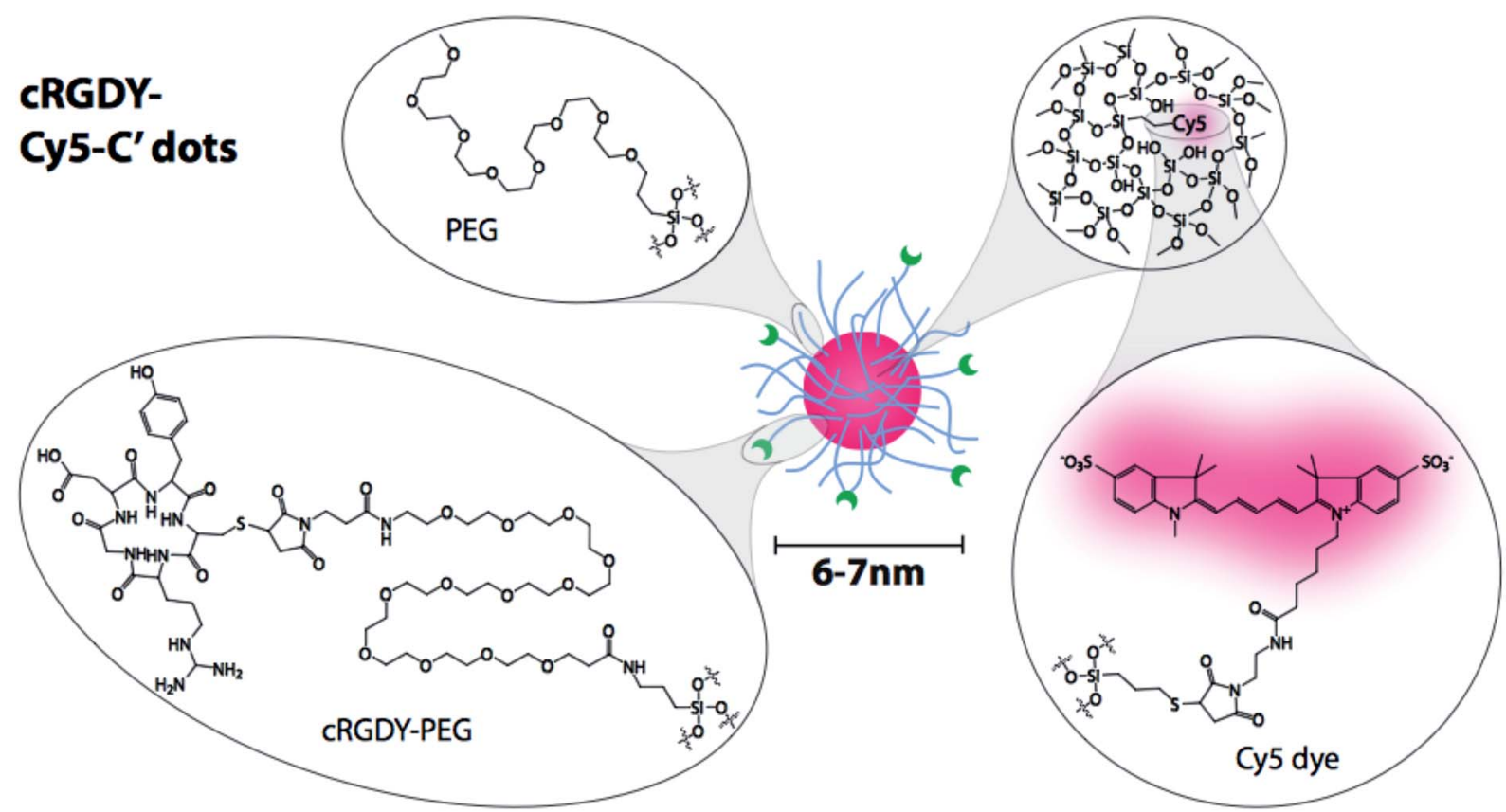

Fig. 1 CycloRGDyC-Cy5-C' dots. These particles are comprised of four components: (1) the cycloRGDyC ligand; (2) PEG oligomers; (3) a silica core; (4) embedded NIR dye (Cy5).

fluorescence correlation spectroscopy (FCS) to simultaneously obtain information on hydrodynamic size, particle concentration, and relative particle brightness, ${ }^{12,14}$ which has helped optimizing synthesis parameters by assessing batch-to-batch variations.

In this report, we introduce the use of RP-HPLC as a tool for $\mathrm{C}^{\prime}$ dot analysis. RP-HPLC has been used to a limited capacity for nanoparticle characterization, ${ }^{19-21}$ as most nanoparticle systems are incompatible due to their large size or relative instability (particles formed by self-assembly or aggregation, e.g. liposomes). We reasoned that $\mathrm{C}^{\prime}$ dots - hybrid particles that exhibit good stability, UV-vis (as well as fluorescence) activity, and are in the size regime of proteins - may be compatible with analytical HPLC approaches. Three detection systems are used for particle analysis: a diode array detector (DAD), an evaporative light scattering detector (ELSD), and an electrospray ionization-single quadrupole mass detector (ESI-SQD). In addition to UV-vis absorption afforded by an embedded Cy5 dye (Fig. 1), the ELSD will provide detection of non-UV active material, such as free PEG or silica-based components. While we do not anticipate mass detection of particles by the ESI-SQD, it was used to monitor the presence of lower molar mass impurities. In addition we describe a new approach to directly estimate the number of surface ligands (cycloRGDyC) on the particle through the use of organic thiols.

\section{Experimental}

\section{Analytical RP-HPLC analysis of $\mathrm{C}^{\prime}$ dots}

CycloRGDyC-Cy5-C ${ }^{\prime}$ dots were synthesized as described elsewhere. ${ }^{10}$ Samples were analyzed on a Waters Alliance HPLC System or Autopure LCMS System (2767 Sample Manager, 2996
Photodiode Array Detector, 2420 ELS Detector, Micromass ZQ, 2525 Binary Gradient Module, Column Fluidics Organizer, 515 HPLC Pump, Pump Control Module II) using a linear gradient of $5-95 \%$ acetonitrile $(\mathrm{ACN})$ in water with $0.05 \%$ trifluoroacetic acid (TFA) or formic acid (FA) for 10 minutes at $1.2 \mathrm{~mL} \mathrm{~min}^{-1}$ on a $\mathrm{C} 184.6 \times 50 \mathrm{~mm}, 300 \AA$ \& $5 \mu \mathrm{m}$ reversed phase XBridge analytical column (Waters). Slower gradients runs were performed using a linear gradient of 5-95\% ACN for $90 \mathrm{~min}$. Samples were analyzed at $280 \mathrm{~nm}$ or $650 \mathrm{~nm}$. $15 \mu \mathrm{M} \mathrm{C^{ \prime }}$ dot stocks (in water) were diluted as needed for RP-HPLC analyses, and the injection volume used was $20-50 \mu \mathrm{L}$.

\section{Thiol-mediated release assay}

For a typical assay, $1 \mu \mathrm{L}$ of $1 \mathrm{M}$ DTT was added to $50 \mu \mathrm{L}$ of $15 \mu \mathrm{M}$ $\mathrm{C}^{\prime}$ dots and incubated at room temperature or $37^{\circ} \mathrm{C}$ overnight. To test glutathione, $25 \mu \mathrm{L}$ of glutathione stock $(100 \mathrm{mM})$ was added to $50 \mu \mathrm{L}$ of $15 \mu \mathrm{M} \mathrm{C}^{\prime}$ dot and incubated overnight. The reaction was analysed by analytical HPLC. The released cycloRGDyC concentrations were determined by comparing peak integrations with a standard curve generated using unconjugated cycloRGDyC peptide.

\section{Results and discussion}

\section{HPLC analysis of $\mathrm{C}^{\prime}$ dots}

For our initial studies, we sought to assess the overall compatibility of $\mathrm{C}^{\prime}$ dots in an analytical RP-HPLC system. We reasoned that the $\sim 6 \mathrm{~nm}$ diameter of the particle is analogous to a globular protein, so a reversed phase C18 column with a $300 \AA$ pore size was selected along with a standard linear gradient of 5-95\% ACN in water (with $0.05 \%$ TFA). As shown in Fig. 2, the particle 
a
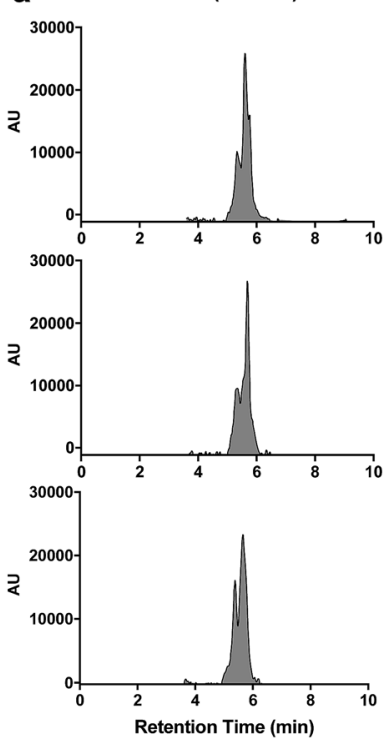

b
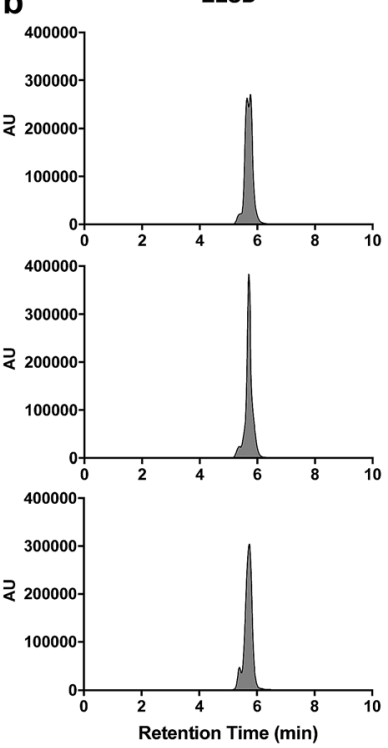

Fig. 2 RP-HPLC chromatograms of cyclo(RGDyC)-Cy5-C' dots, 1. (a) DAD at $650 \mathrm{~nm}$; (b) ELSD. Three consecutive analyses of the same sample (triplicate). $1.5 \mu \mathrm{M}$ particle concentration; $20 \mu \mathrm{L}$ injection volume; chromatographic conditions were $5-95 \%$ ACN $(0.05 \%$ TFA) in $10 \mathrm{~min}$.

elutes as a broad signal containing multiple peaks when monitored at $650 \mathrm{~nm}\left(\mathrm{C}^{\prime}\right.$ dots contain the near-infrared-dye Cy5, which exhibits a characteristic absorption maximum at $650 \mathrm{~nm}$ ) and by the ELSD. Interestingly, peak shapes changed from runto-run.

When samples were analyzed in triplicate, the overall retention times were similar, but peak shapes differed significantly between each run (Fig. 2). Remarkably, peak integrations proved to be very consistent, with relative standard deviations (RSD) of 7\%, suggesting excellent run-to-run reproducibility (Table 1). Similar results were observed when monitored by the ELSD - peak profiles varied significantly from run-to-run, whereas peak integrations showed excellent consistency $(\mathrm{RSD}=5 \%)$ (Fig. 3b, Table 1).

To further highlight the consistency of the RP-HPLC for $\mathrm{C}^{\prime}$ dot analysis, a nanoparticle concentration curve was generated. Particle samples of varying concentrations ranging from $1.5 \mu \mathrm{M}$ to $8 \mu \mathrm{M}$ were analyzed and peak integrations at $650 \mathrm{~nm}$ where determined and plotted against concentrations. As shown in Fig. 3A, an excellent linear response was observed with the DAD $\left(R^{2}=0.9986\right)$. Even concentrations down to $0.5 \mu \mathrm{M}$ and up to $60 \mu \mathrm{M}$ (not shown) were also in the linear range of the detector. Unexpectedly, the ELSD response also exhibited excellent

Table 1 Peak integrations for three identical RP-HPLC runs

\begin{tabular}{lcccc}
\hline & \multicolumn{1}{l}{ Run } & & & \\
\cline { 2 - 5 } & 1 & 2 & 3 & \pm RSD (\%) \\
\hline DAD & 11698 & 10218 & 11426 & 7 \\
ELSD & 85467 & 78326 & 86755 & 5
\end{tabular}
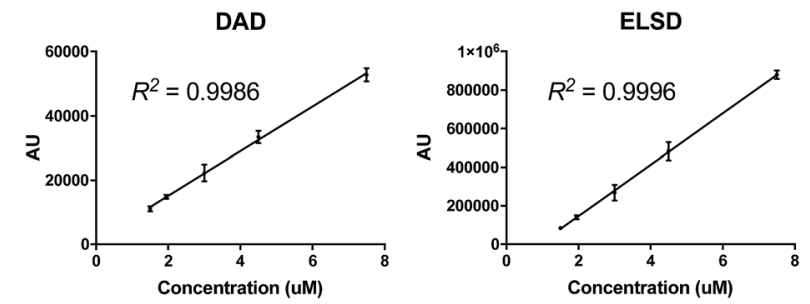

Fig. 3 Peak areas versus particle concentration. Five particle concentrations $(1.5,2.0,3.0,4.5,8.0 \mu \mathrm{M})$ were analyzed by RP-HPLC (triplicate). $20 \mu \mathrm{L}$ injection volume; chromatographic conditions were $5-95 \%$ ACN $(0.05 \%$ TFA) in $10 \mathrm{~min}$. Chromatogram peaks from the DAD $(650 \mathrm{~nm})$ and ELSD were integrated and plotted verses concentration.

linearity in the $1.5-8 \mu \mathrm{M}$ range $\left(R^{2}=0.9996\right)$. In summary, both detectors provide consistent linearity in the $\sim 1-10 \mu \mathrm{M}$ concentration range.

The peak shapes seen in the HPLC chromatograms indicate a heterogeneous mixture of compounds. To further investigate the nature of the observed peaks, nanoparticle samples were analyzed using a slower or more shallow gradient. While initial analyses (Fig. 2) were performed at 5-95\% ACN in $10 \mathrm{~min}$ (gradient of $9 \%$ ACN per min), Fig. 4 shows chromatograms run at $5-95 \% \mathrm{ACN}$ in water for $90 \mathrm{~min}$, which translates into a gradient of $1 \%$ ACN per min. Under these conditions, the broad peaks as evident in Fig. 2, are better resolved, revealing numerous peaks. However, once again, run-to-run variations in

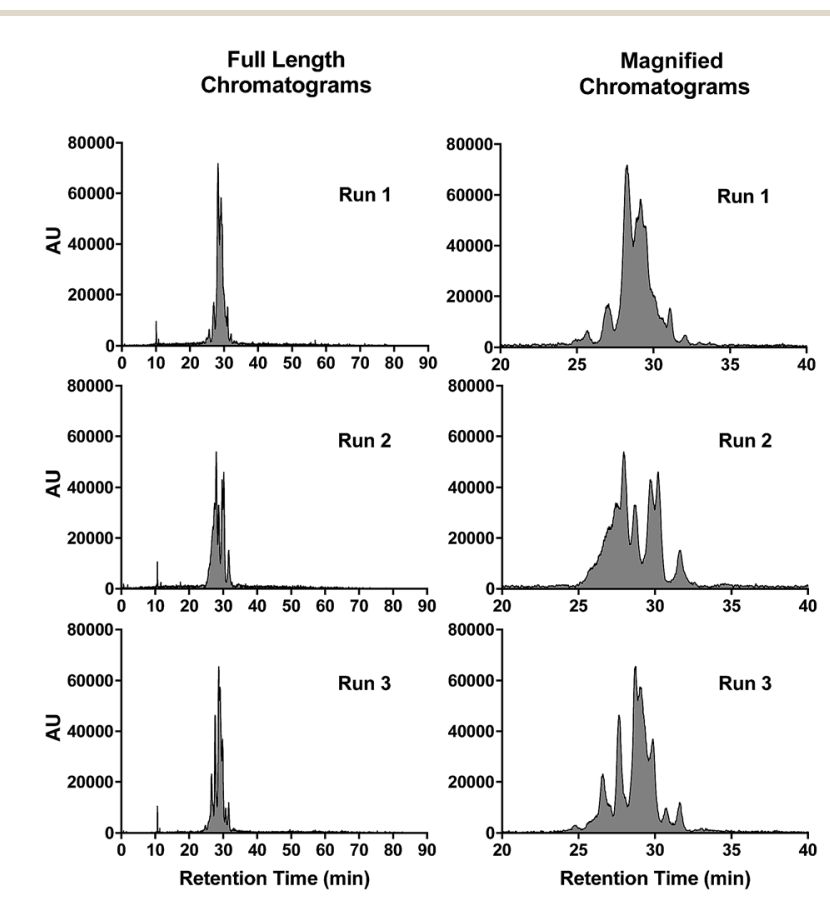

Fig. 4 RP-HPLC chromatograms of cycloRGDyC-C' dot using a slower gradient. Three consecutive analyses of the same sample (triplicate) were performed. Panels on the left show the full-length chromatograms (0-90 $\mathrm{min})$; panels on the right show a magnified portion of the chromatograms (20-40 min). RP-HPLC conditions were $5-95 \%$ ACN $(0.05 \%$ TFA) in $90 \mathrm{~min}$; DAD at $650 \mathrm{~nm}$. 
peak profiles are observed. Yet remarkably, the peak integrations at $650 \mathrm{~nm}$ demonstrate excellent consistency $(\mathrm{RSD}=$ $5.6 \%)$.

Possible explanations for the changing peak shapes are that the particle is chemically degrading or undergoing dynamic changes in surface charge or PEG conformations. Interestingly, $\mathrm{C}^{\prime}$ dots containing no cycloRGDyC peptide also exhibit the same changing peak profiles (data not shown). This suggests that the peptide ligands are not the main contributors to the observed peak features. The HPLC analyses are run under acidic conditions, so one possibility would be that the particles are being slowly hydrolyzed. However, cycloRGDyC-Cy5-C' dots subjected to acidic RP-HPLC conditions (0.05\% TFA in water) over time (1-16 hours), showed no distinction with regard to peak shape, retention time, or peak integrations (data not shown). Also, when these particles are analyzed by GPC, elution volumes were identical to non-treated particles. Weaker acid conditions (0.05\% FA in water) showed no effect, as RP-HPLC chromatograms were consistent with TFA runs.

The run-to-run variations in peak profiles may be explained by the particle undergoing dynamic changes in surface charge and PEG conformations. This is consistent with our observations that the zeta-potential of $\mathrm{C}^{\prime}$ dots is never exactly zero, but always slightly negative. ${ }^{10}$ Additionally, the remaining free silanol groups on the $\mathrm{C}^{\prime}$ dot surfaces can undergo intramolecular interactions with the PEG groups. For example, the formation of hydrogen bonds between the ether oxygen of PEGs and silicic acid has been described. ${ }^{\mathbf{1 3 , 2 2}}$ Taken together, this suggests that the particles are stable under HPLC conditions, and the changing peak profiles may be due to the dynamic nature of the PEG surface layer.

\section{Thiol-mediated release of ligands}

While exposing the cycloRGDyC-Cy5-C $\mathrm{C}^{\prime}$ dots to various in vitro assay conditions and monitoring by RP-HPLC, we observed an unexpected phenomenon where the cycloRGDyC could be released in the presence of commonly used small organic thiols such as dithiothreitol (DTT). In the synthesis of the nanoparticle, cycloRGDyC is attached to heterobifunctional PEGs through a thiol-maleimide linkage (Fig. 5). ${ }^{10}$ Recently it has

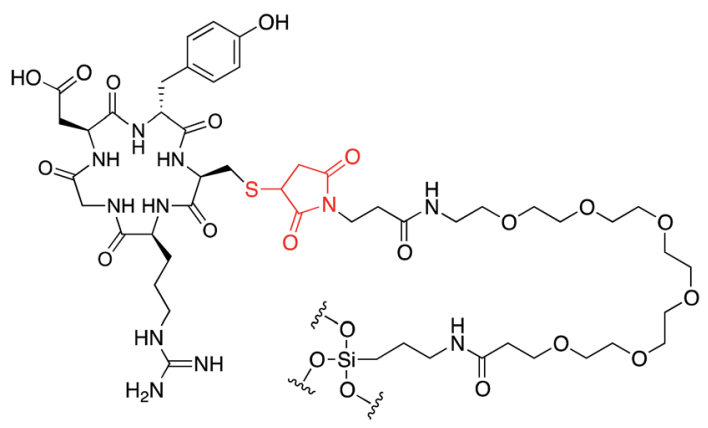

Fig. 5 Cyclo(RGDyC) on $C^{\prime}$ dots. The cyclo(RGDyC) peptide is covalently attached to the particle through a cysteine-maleimido-PEG-silyl group. The thiol-maleimide group (red) may be susceptible to a reverse Michael reaction. been shown in the context of antibody drug conjugates that thiol-maleimides can be susceptible to reverse Michael reactions in the presence of other intermolecular thiols. These studies have shown this reaction to be highly sequence dependent on antibodies. To assess the stability of the thiolmaleimide linkage on the $\mathrm{C}^{\prime}$ dots, the particles were subjected to thiol-containing compounds DTT or glutathione for $24 \mathrm{~h}$ at $37^{\circ} \mathrm{C}$, then analyzed by LCMS (glutathione is ubiquitous in vivo tripeptide containing cysteine). Under these conditions we observed efficient release of the cycloRGDyC when DTT was present. However minimal release $(<5 \%)$ was observed with glutathione (data not shown) at up to 48 hours. Furthermore, $\mathrm{C}^{\prime}$ dots incorporating different amounts of the ligand could be assessed using this method. Typically, ligand quantitation is estimated by UV absorption spectroscopy. ${ }^{10}$ Three $\mathrm{C}^{\prime}$ dots, estimated to contain on average 6, 14, or 18 cycloRGDyC peptides by UV, were treated with DTT and analyzed by RPHPLC (Fig. 6A). A standard curve with the unconjugated cycloRGDyC peptide was also generated. Peak integrations of the released peptides from the 6,14 and 18 cycloRGDyC-Cy5-C' $\mathrm{C}^{\prime}$ dot samples were obtained; and their corresponding concentrations and ligand per particle values were determined from the standard curve. Fig. 6B shows a plot comparing the levels of cycloRGDyC per particles as estimated by UV spectroscopy verses this thiol-mediated release assay, and good correlation is observed between these two methods. In addition to estimating ligand number or concentration, thiol-mediated release may provide insight into the spatial location of the ligands. Given the accessibility of the DTT to the maleimides, and subsequent

\section{A Thiol-Mediated Release of CycloRGDyC}

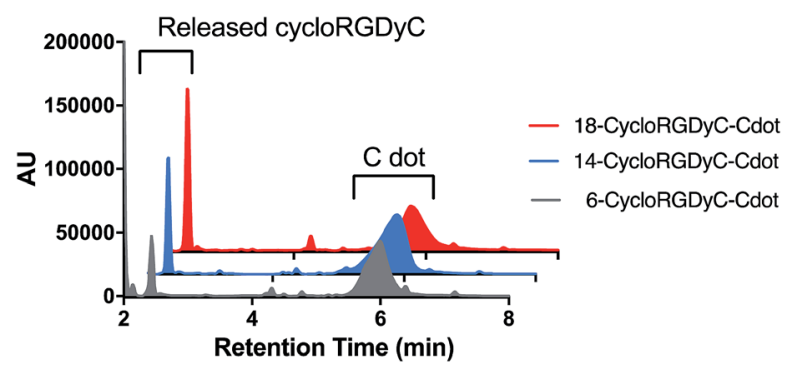

B Thiol-Mediated Release vs. Absorbance

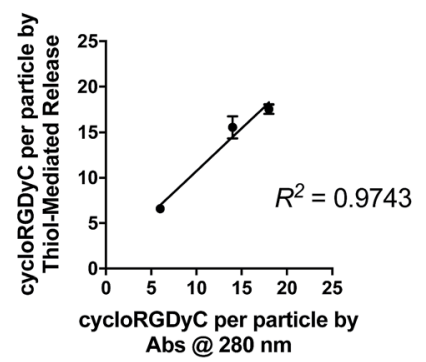

Fig. 6 Thiol-mediated release of cycloRGDyC from $C^{\prime}$ dots. (A) $C^{\prime}$ dots containing different numbers of cycloRDyC (6, grey; 14 , blue; and 18 , red) are treated with DTT then analyzed by RP-HPLC. (B) Plot comparing cycloRGDyC per particle estimates from UV absorbance verses thiol-mediated release. 
release of the peptides, this supports the notion that the peptide is likely near or on the surface of the particle, and not embedded within, consistent with the schematic in Fig. 1.

\section{Conclusions}

In this report, we expand the use of traditional analytical tools for the characterization of inorganic-organic hybrid nanoparticles to high resolution HPLC. In particular, we employ two RP-HPLC based analytical approaches for a specific type of ultrasmall silica based nanoparticles called $\mathrm{C}^{\prime}$ dots. As the clinical applications of these nanoparticles expand, such new approaches are needed to refine characterization and assessment of their exact composition and structure. While detailed peak shapes were inconsistent from run-to-run, which could represent different charged or conformational states of the PEGylated particle surface; other features were found to be highly reproducible, such as retention time and peak integrations, providing an overall predictable and linear response. Further optimization with solvent conditions may be needed (i.e. the use of different solvent additives such as buffers, salts or ion pairing reagents) and is currently ongoing. However, the data suggests that RP-HPLC may provide a high resolution means of separating distinct subpopulations of nanoparticles. An unexpected phenomenon was observed where cysteinemaleimides incorporated onto the particles were susceptible to low molar mass thiols, but not glutathione. Excess DTT was used to release cycloRGDyC from the $\mathrm{C}^{\prime} \mathrm{dot}$, and released peptide could be measured to obtain ligand to particle estimates.

\section{Acknowledgements}

The authors gratefully acknowledge Dr Feng Chen for his thoughtful insights and suggestions; and support by the National Cancer Institute of the National Institutes of Health under Award Number U54CA199081. We would like to further acknowledge the NMR Core Facility at MSKCC and Hunter Mass Spectrometry for use of their instrumentation. This work made use of the Cornell Center for Materials Research shared facilities supported through the NSF MRSEC program (DMR1120296), and the Nanobiotechnology Center shared research facilities at Cornell.

\section{Notes and references}

1 C. M. Dawidczyk, C. Kim, J. H. Park, L. M. Russell, K. H. Lee, M. G. Pomper and P. C. Searson, J. Controlled Release, 2014, 187, 133-144.

2 A. Ediriwickrema and W. M. Saltzman, ACS Biomater. Sci. Eng., 2015, 1, 64-78.
3 A. Eifler and C. S. Thaxton, in Biomedical Nanotechnology, ed. S. J. Hurst, Humana Press, 2011, vol. 726, ch. 21, pp. 325338.

4 T. Sun, Y. S. Zhang, B. Pang, D. C. Hyun, M. Yang and Y. Xia, Angew. Chem., Int. Ed., 2014, 53, 12320-12364.

5 E. Phillips, O. Penate-Medina, P. B. Zanzonico, R. D. Carvajal, P. Mohan, Y. Ye, J. Humm, M. Gönen, H. Kalaigian, H. Schöder, H. W. Strauss, S. M. Larson, U. Wiesner and M. S. Bradbury, Sci. Transl. Med., 2014, 6, 260 ra149.

6 D. Bobo, K. J. Robinson, J. Islam, K. J. Thurecht and S. R. Corrie, Pharm. Res., 2016, 33, 2373-2387.

7 E. J. Cho, H. Holback, K. C. Liu, S. A. Abouelmagd, J. Park and Y. Yeo, Mol. Pharm., 2013, 10, 2093-2110.

8 Y. Piao, A. Burns, J. Kim, U. Wiesner and T. Hyeon, $A d v$. Funct. Mater., 2008, 18, 3745-3758.

9 P.-C. Lin, S. Lin, P. C. Wang and R. Sridhar, Biotechnol. Adv., 2014, 32, 711-726.

10 K. Ma, C. Mendoza, M. Hanson, U. Werner-Zwanziger, J. Zwanziger and U. Wiesner, Chem. Mater., 2015, 27, 41194133.

11 A. Lopez-Serrano, R. M. Olivas, J. S. Landaluze and C. Camara, Anal. Methods, 2014, 6, 38-56.

12 H. Ow, D. R. Larson, M. Srivastava, B. A. Baird, W. W. Webb and U. Wiesner, Nano Lett., 2005, 5, 113-117.

13 K. Ma, D. Zhang, Y. Cong and U. Wiesner, Chem. Mater., 2016, 28, 1537-1545.

14 D. R. Larson, H. Ow, H. D. Vishwasrao, A. A. Heikal, U. Wiesner and W. W. Webb, Chem. Mater., 2008, 20, 2677-2684.

15 E. Herz, H. Ow, D. Bonner, A. Burns and U. Wiesner, J. Mater. Chem., 2009, 19, 6341-6347.

16 M. Benezra, O. Penate-Medina, P. B. Zanzonico, D. Schaer, H. Ow, A. Burns, E. DeStanchina, V. Longo, E. Herz, S. Iyer, J. Wolchok, S. M. Larson, U. Wiesner and M. S. Bradbury, J. Clin. Invest., 2011, 121, 2768-2780.

17 A. A. Burns, J. Vider, H. Ow, E. Herz, O. Penate-Medina, M. Baumgart, S. M. Larson, U. Wiesner and M. Bradbury, Nano Lett., 2008, 9, 442-448.

18 B. Yoo, K. Ma, L. Zhang, A. Burns, S. Sequeira, I. Mellinghoff, C. Brennan, U. Wiesner and M. S. Bradbury, Bioorg. Med. Chem., 2015, 23, 7119-7130.

19 R. A. González-Fuenzalida, Y. Moliner-Martínez, C. MolinsLegua, V. Parada-Artigues, J. Verdú-Andrés and P. Campins-Falcó, Anal. Chem., 2016, 88, 1485-1493.

20 S. Xie, M. C. Paau, Y. Zhang, S. Shuang, W. Chan and M. M. F. Choi, Nanoscale, 2012, 4, 5325-5332.

21 C. Truillet, F. Lux, O. Tillement, P. Dugourd and R. Antoine, Anal. Chem., 2013, 85, 10440-10447.

22 M. Preari, K. Spinde, J. Lazic, E. Brunner and K. D. Demadis, J. Am. Chem. Soc., 2014, 136, 4236-4244. 